\title{
1,4-双[2-(4-吡啶基)乙烯基]苯衍生物的合成、光谱性质及理论研究
}

\author{
欧亚平 $*, a$ 张 静 $b$ 庾江喜 $a$ 朱小明 $a$

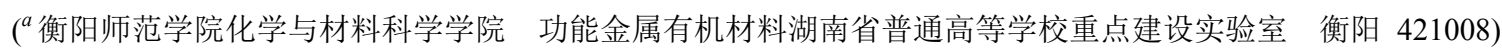 \\ $(b$ 华中师范大学化学学院 农药与化学生物学教育部重点实验室 武汉 430079)
}

\begin{abstract}
摘要 通过经典的 Witting-Horner 反应及 Sonogashira 交叉偶联反应以较高的产率合成得到 4 个不对称型氢键受体分子 1,4-双[2-(4-吡啶基)乙烯基]苯衍生物, 并对其结构及光谱性质开展了研究. 系列化合物均通过核磁、元素分析及质谱的 表征, 晶体结构表明由于苯环邻位芳环乙炔基的空间效应导致氢键组装的构型发生改变. 紫外可见光谱结合含时密度 泛函理论(TDDFT)计算表明目标化合物在紫外区域均呈现出强烈的 $\pi \rightarrow \pi^{*}$ 及 ICT 跃迁吸收. 苂光光谱表明随着共轭体 系的逐渐增大也导致了它们的发射峰有一个红移的倾向，且有利于分子之间形成 $\pi-\pi$ 堆积. 以上结果将为后续的氢键 自组装的区域选择性及光照环化实验研究提供了一定的合成基础及理论依据.
\end{abstract}

关键词 不对称氢键受体分子; 1,4-双[2-(4-吡啶基)乙烯基]苯; 晶体结构; 光谱性质

\section{Synthesis, Spectral Properties and Theoretical Studies of 1,4-Bis[2-(4-pyridyl)ethenyl]benzene Derivatives}

\author{
$\mathrm{Ou}$, Yaping ${ }^{*, a}$ \\ Zhang, Jing $^{b}$ \\ Yu, Jiangxi ${ }^{a}$ \\ Zhu, Xiaoming ${ }^{a}$ \\ $\left({ }^{a}\right.$ Key Laboratory of Functional Organometallic Materials of Hunan Province College, College of Chemistry and Material \\ Science, Hengyang Normal University; Hengyang 421008) \\ $\left({ }^{b}\right.$ Key Laboratory of Pesticide \& Chemical Biology, Ministry of Education, Central China Normal University, Wuhan 430079)
}

\begin{abstract}
Four asymmetric hydrogen-bond acceptors of 1,4-bis[2-(4-pyridyl)ethenyl]benzene derivatives with higher yields were synthesized through the classical Witting-Horner and Sonogashira cross-coupling reactions, and their structures and spectral properties were investigated. The series of compounds all have been characterized by NMR, MS and element analysis. Crystal structures indicated that the conformation of hydrogen-bond self-assemble may be change due to the hindrance effect from the arylacetyl group in $o$-position of benzene. UV-Vis spectra combined with time-dependent density functional theory (TDDFT) calculation results showed that target compounds all display a strong $\pi \rightarrow \pi^{*}$ and ICT transition absorptions. Fluorescence spectrum indicated that gradual increasing of conjugate systems make their emission peak tend to a red shift, which is conducive to $\pi-\pi$ accumulation. Above results will provide certain synthetic basis and theoretical foundation for subsequent studies about regioselectivity of hydrogen-bond self-assemble and cyclization reactions from light.
\end{abstract}

Keywords Asymmetric hydrogen-bond acceptor; 1,4-Bis[2-(4-pyridyl)ethenyl]benzene; Crystal structure; Spectral properties

1987 年, C. J. Pedersen(佩德森)、J. M. Lehn(莱恩)、 D. J. Cram(克来姆)三位化学家被授予诺贝尔化学奖以 表彰他们在超分子化学领域的杰出贡献, 自此以后, 超 分子化学作为一门新兴的科学快速蓬勃发展起来, 并以 其强大的生命力和广阔的应用前景逐渐发展成为许多 科学家们的主要研究领域和前沿课题. 超分子化学主要 研究的是弱相互作用(即非共价作用), 如氢键作用、 $\pi-\pi$
作用、疏溶剂作用、偶极作用、静电作用即范德华力等. 其内容更为丰富，主要有分子识别、分子自组装、超分 子光化学、超分子催化以及超分子与生物科学相结合等, 目前已经渗透到生物、环境、医学、材料、农业等众多 学科领域 ${ }^{[1 \sim 4]}$.

分子自组装是超分子化学的一个重要研究内容. 所 谓分子自组装，即分子与分子之间通过弱相互作用(非

* Corresponding author. E-mail: ouyaping@hynu.edu.cn

Received June 29, 2016; revised September 2, 2016; published online October 18, 2016.

Project supported by the Opening Subjects of Key Laboratory of Functional Organometallic Materials of Hunan Province (No. GN15K05), and the Scientific Research Foundation of the Hengyang Normal University (No. 14B23).

湖南省高校重点实验室开放基金(No. GN15K05)及衡阳师范学院科研启动基金(No. 14B23)资助项目. 
共价作用)自发形成的稳定的分子聚集体过程, 其本质 就是分子识别作用, 在生命体系中普遍存在, 许多复杂 且有生物学功能的超分子系统如蛋白质、核酸等都是通 过分子自组装形成的 ${ }^{[5]}$. 特别的是, 它也作为合成一些 分子的重要手段, 可以合成常规合成手段无法得到的化 合物 ${ }^{[6-7]}$. MacGillivray 等 ${ }^{[8 \sim 12]}$ 已经在这方面做了大量的 研究工作, 目的是利用这种弱相互作用(非共价作用)来 指导共价键的形成, 尤其通过小的有机分子或者金属有 机配合物作为模版分子与反应物(一般为刚性的烯烃分 子)经过分子识别和自组装来诱导固态(共晶)条件下符 合光化学活性的 $[2+2]$ 环加成反应, 如图 1 所示. 该方 法能够合成不同立体构型的四元环状化合物且具有较 高的产率, 得到的环丁烷衍生物可以作为天然产物分子 的一个重要构建单元以及其他反应的合成子. 同时模版 分子可以回收再利用, 这样为有机合成策略提供了一条 新途径.

分子自组装后共晶, 在固态下发生 $[2+2]$ 环加成反
应必须要达到 Schmidt 光致环化条件. 那么这依赖于模 版分子的选择以及反应物本身的性质，通常所用的模版 分子有(含金属有机模版)几种 ${ }^{[8]}$, 如图 2 所示. 也正是由 于模版分子的不同从而可以控制两分子中双键之间的 距离. 此外, 通过研究肉桂酸在固态下发生光致环化的 条件发现, 烯烃发生 $[2+2]$ 环加成两个双键处在平行的 位置且它们之间的距离应小于 $4.2 \AA$, 如图 3 所示. 由此 可见在固态条件下, 只要分子在堆积形态下的双键距离 小于 $4.2 \AA$ 且处于平行的位置, 那么在光照条件下就可 能发生环加成反应.

随着研究的不断深入, 科学家们不断研究影响分子 自组装、共晶以及光致环化的各种因素, 从而有利于多 元环状化合物的合成, 2003 年, MacGillivray 等 ${ }^{[13]}$ 通过间 苯二酚衍生物为模版与反应物 1,4-双[2-(4-吡啶基)乙烯 基]苯进行自组装，然后共晶，固态条件下再经过光照 关环，脱去模版分子得到了 [2.2]对环芳衍生物，如图 4 所示. [2.2]对环芳结构单元本身就作为一个具有面面堆

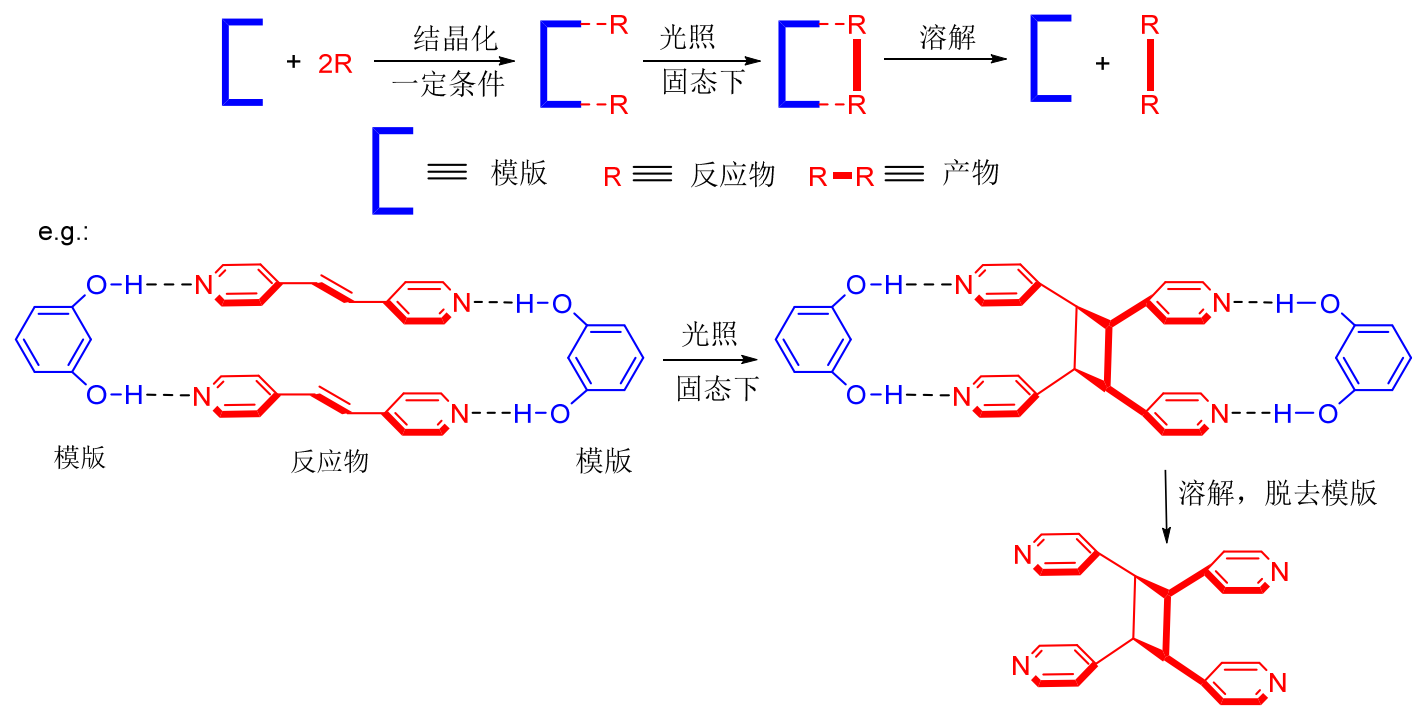

图 1 模版分子与反应物分子自组装及 $[2+2]$ 环加成反应过程

Figure 1 Self-assembly of template and reactant molecular and process of $[2+2]$ photodimerizations

有机分子

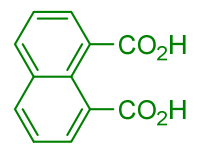<smiles>c1ccc2cc(CSc3ccncc3)c(CSc3ccncc3)cc2c1</smiles>

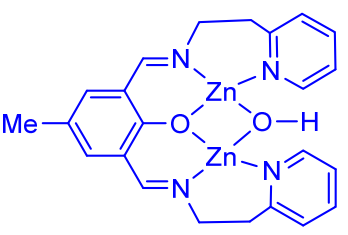

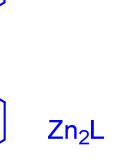

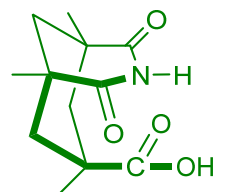

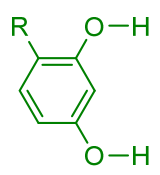

金属有机配合物

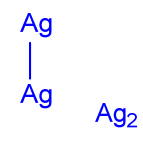

图 2 不同类型的模版分子(有机小分子及金属模版)

Figure 2 Different templates molecules (small organic molecular and metal-organic templates) 


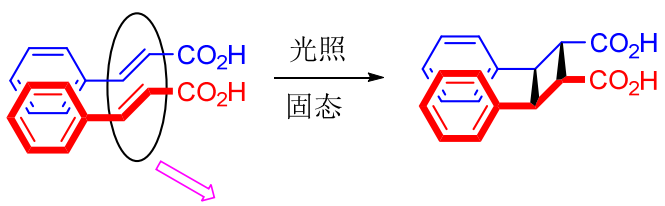

处于平行的位置, 距离 $<4.2 \AA$

图 3 分子在固态条件下发生光致环化的条件

Figure 3 Conditions of photodimerization to molecular in the solid state

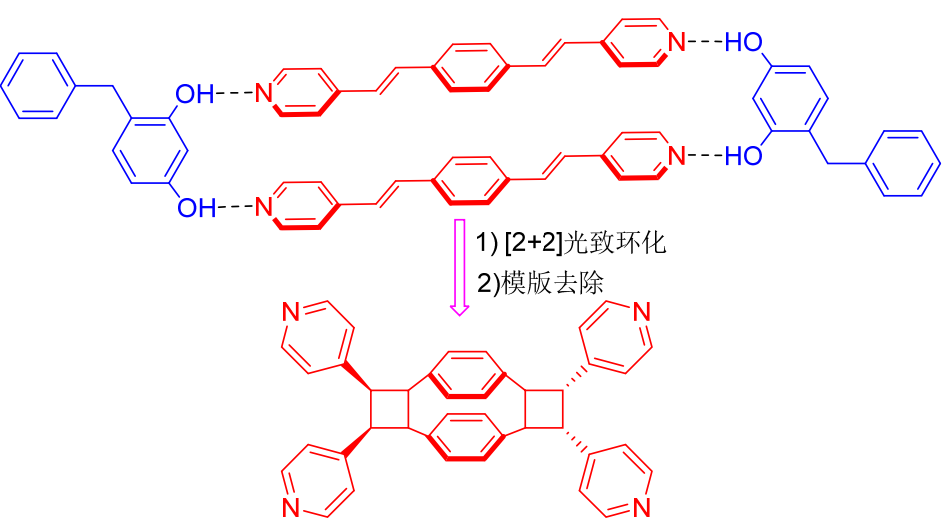

图 4 1,4-双[2-(4-吡啶基)乙烯基]苯与间苯二酚衍生物共晶光照下固态关环形成[2.2]对环芳衍生物

Figure 4 Cocrystallization of 1,4-bis[2-(4-pyridyl)ethenyl]benzene with resorcinol derivatives, and forming the [2.2]paracyclophane derivatives by UV-irradiation

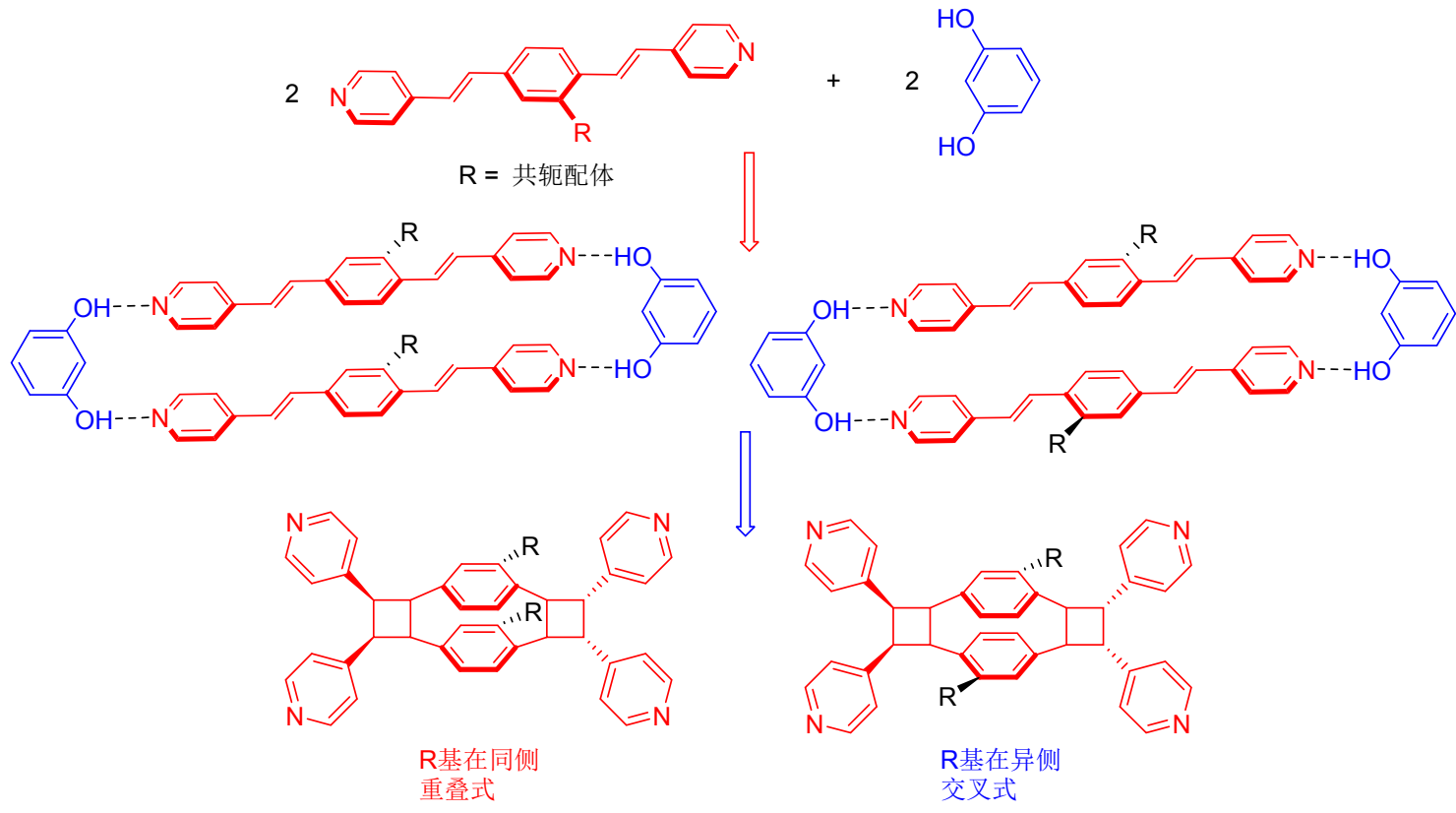

图 5 不对称氢键受体分子与间苯二酚两种氢键自组装情况及两种不同的光致环化产物

Figure 5 Hydrogen-bond self-assemble situations of asymmetric hydrogen-bond acceptor with resorcinol and two different photodimerization products

积作用的例子，其衍生物已广泛应用于不对称合成、生 物医药、纳米技术以及催化等 ${ }^{[14 ~ 17]}$, 因此该方法对[2.2] 对环芳衍生物的合成提供了一条新的途径.

通过文献调研发现, 以上的分子自组装主要驱动力 是氢键作用及 $\pi-\pi$ 作用, 且氢键受体分子均是对称的乙
烯基吡啶衍生物，如 1,4-双[2-(4-吡啶基)乙烯基]苯，如 果在其中间的苯环上引入具有共轭体系的取代基，那么 两分子氢键受体与两分子氢键给体在进行自组装时, 可 能会出现两种情况(交叉式和重叠式)(如图 5 所示), 产 生了区域选择性, 然后经过强紫外光照, 分子间平行双 
键之间发生关环，从而合成具有区域选择性的[2.2]对环 芳衍生物. 究竟出现那种自组装形式, 依赖于取代基之 间 $\pi-\pi$ 堆积作用强弱、电子性质以及空间效应等因素.

基与以上分析, 为了探索氢键组装下, $\pi-\pi$ 堆积作 用、电子效应及空间效应所形成的区域选择性组装情况, 本论文特设计合成如图 6 所示一系列不对称的氢键受体 分子 1,4-双[2-(4-吡啶基)乙烯基]苯衍生物, 并对其结构 及性质进行表征. 希望为后续探索区域选择性自组装情 况提供合成基础和理论依据.
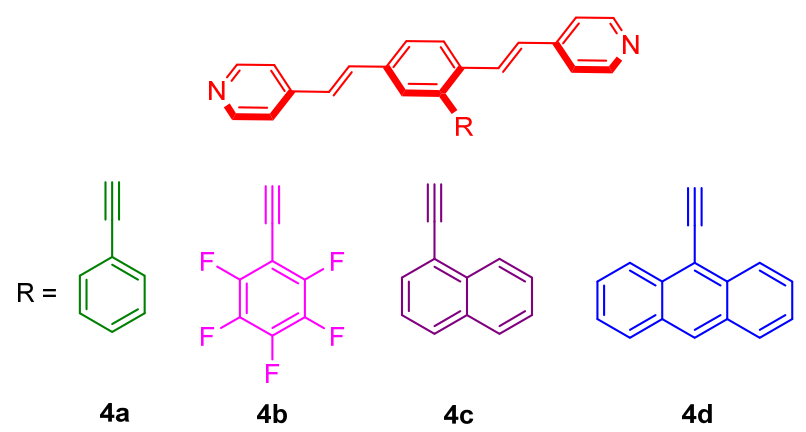

图 6 不对称的氢键受体分子 1,4-双[2-(4-吡啶基)乙烯基]苯衍 生物 $\mathbf{4 a} \sim \mathbf{4 d}$

Figure 6 Asymmetric hydrogen-bond acceptor 1,4-bis[2-(4pyridyl)ethenyl]benzene derivatives $\mathbf{4 a} \sim \mathbf{4 d}$

\section{1 结果与讨论}

\section{1 目标化合物的合成}

目标化合物的合成经历了两个重要的人名反应, 即 Witting-Horner 反应及 Sonogashira 交叉偶联反应, 其合 成路线如图 7 所示.

中间体 2 通过类似文献[20]的方法以 70\%的收率合 成得到, 其在常温下为粘稠液体, 若将其置于冰箱冷冻, 则为白色固体. 从中间体 2 至中间体 3 的经典的
Witting-Horner 反应，关键是通过强碱来控制形成双键 的顺反. 在该反应中, 我们选择的强碱是 $\mathrm{KOBu}-t$, 生成 的产物绝大多数为反式产物, 且顺式产物可以通过重结 晶的方法除去，从而以 $65 \%$ 的收率得到中间体 3. 生成 的溴代产物 3 再分别与末端炔(苯乙炔、五氟苯乙炔、 $\alpha$ 菜乙炔及 9-葱乙炔)在 $\mathrm{Pd}\left(\mathrm{PPh}_{3}\right)_{4}$ 及 $\mathrm{CuI}$ 的作用下发生 Sonogashira 交叉偶联反应，分别以中等以上的收率得 到 $4 a \sim 4 d$.

\section{2 目标化合物 $4 \mathrm{~b}$ 的 $X$ 射线晶体结构}

目标化合物 $4 b$ 经过了 $X$ 射线单晶衍射表征, 该晶 体是通过扩散法(氯仿/甲醇)在室温下培养(放置近 $30 \mathrm{~d}$ 后)得到. 其为棕黄色块状晶体. 化合物 $\mathbf{4 b}$ 的分子结构 图如图 8 所示, 对应的部分键长、键角及二面角被列在 表 1 中. 从键长数据中可以看出, $\mathrm{C}(14)-\mathrm{C}(15)$ 及 $\mathrm{C}(21)-\mathrm{C}(22)$ 的键长各为 1.292 和 $1.191 \AA$, 分别对应于 碳碳双键及参键键长范围. $C(6)-C(7)-C(8)$ 键角接近 $120^{\circ}$, 也可以很明显看出形成的为反式双键, $C(10)$ $\mathrm{C}(21)-\mathrm{C}(22)$ 键角接近 $180^{\circ}$, 也进一步证实了参键的存 在. 平面 $[C(9), C(10), C(21)]$ 与平面 $[C(22), C(23), C(28)]$ 之间的夹角接近于 $0^{\circ}$, 表明该分子是一个高度共平面的 分子，从而使得分子与分子之间可能存在一定的 $\pi-\pi$ 堆 积, 从图 8(b)可以看出这种堆积作用. CCDC 号为 1488285.

\section{3 光谱及理论计算研究}

1.3.1 紫外可见吸收光谱研究及含时密度泛函理论 计算

为了考察目标化合物的电子跃迁情况，目标化合物 4a $\sim \mathbf{4 d}$ 及中间体 3 均被进行了紫外可见光谱测试(浓度 为 $2.0 \times 10^{-5} \mathrm{~mol} / \mathrm{L}$ 的 $\mathrm{CH}_{2} \mathrm{Cl}_{2}$ 溶液), 对应的电子跃迁吸 收光谱如图 9 所示.

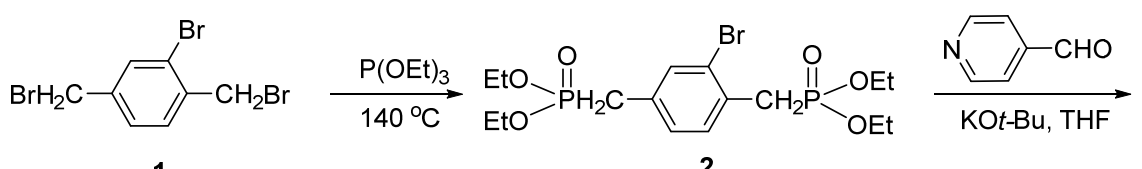

1

2

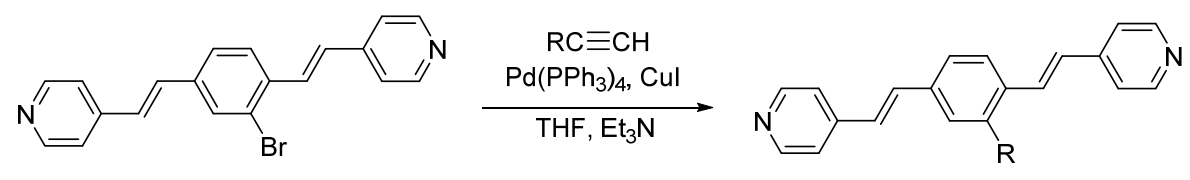

3<smiles>Cc1ccccc1</smiles>

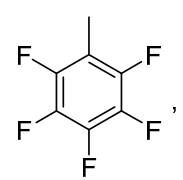

4b

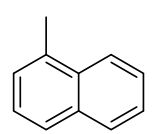

4c

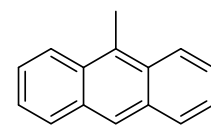

4d

图 7 目标化合物 $\mathbf{4 a \sim 4 d}$ 的合成路线示意图

Figure 7 Synthetic route diagram of target compounds $\mathbf{4 a} \sim \mathbf{4 d}$ 
(a)

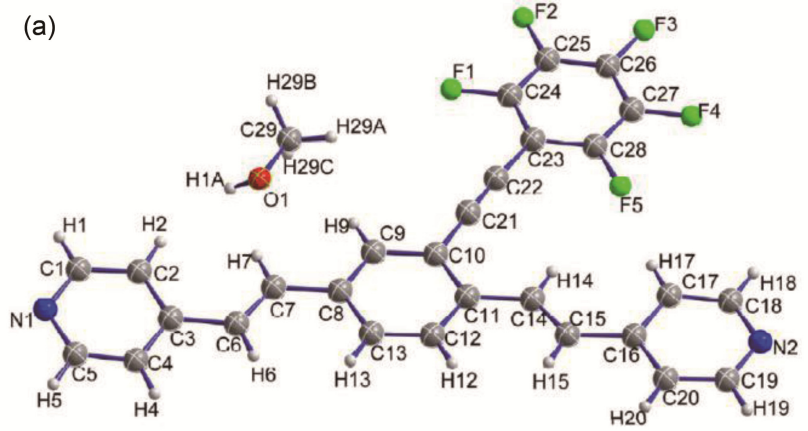

(b)

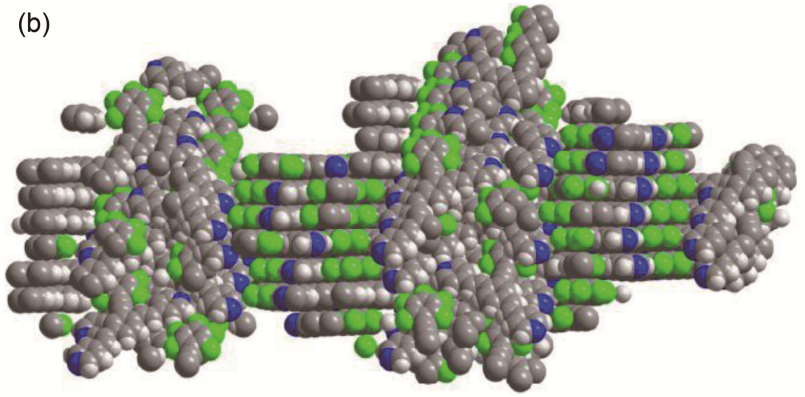

图 8 目标化合物 $\mathbf{4 b}$ 的分子结构图

Figure 8 Molecular structure of target compound $\mathbf{4 b}$ (a) Top view; (b) side view

表 1 目标化合物 $\mathbf{4 b}$ 的晶体结构对应的部分键长 $(\AA)$ 、键角 $\left(^{\circ}\right)$ 和二面角 $\left({ }^{\circ}\right)$

Table 1 Selected bond lengths $(\AA)$, angles $\left(^{\circ}\right)$ and dihedral angle $\left(^{\circ}\right)$ from crystal structure of $\mathbf{4 b}$

\begin{tabular}{|c|c|c|c|}
\hline \multicolumn{4}{|c|}{ 键长/Å } \\
\hline $\mathrm{N}(2)-\mathrm{C}(18)$ & 1.311 & $\mathrm{C}(11)-\mathrm{C}(10)$ & 1.394 \\
\hline $\mathrm{C}(18)-\mathrm{C}(17)$ & 1.370 & $\mathrm{C}(10)-\mathrm{C}(21)$ & 1.442 \\
\hline$C(17)-C(16)$ & 1.384 & $\mathrm{C}(21)-\mathrm{C}(22)$ & 1.191 \\
\hline$C(16)-C(15)$ & 1.489 & $\mathrm{C}(22)-\mathrm{C}(23)$ & 1.426 \\
\hline $\mathrm{C}(15)-\mathrm{C}(14)$ & 1.292 & $\mathrm{C}(23)-\mathrm{C}(28)$ & 1.392 \\
\hline $\mathrm{C}(14)-\mathrm{C}(11)$ & 1.456 & $\mathrm{C}(28)-\mathrm{F}(5)$ & 1.335 \\
\hline \multicolumn{4}{|c|}{ 键角 $/\left(^{\circ}\right)$} \\
\hline \multicolumn{3}{|c|}{$C(6)-C(7)-C(8)$} & 126.52 \\
\hline \multicolumn{3}{|c|}{$C(9)-C(10)-C(21)$} & 119.54 \\
\hline \multicolumn{3}{|c|}{$\mathrm{C}(10)-\mathrm{C}(21)-\mathrm{C}(22)$} & 178.25 \\
\hline \multicolumn{4}{|c|}{ 二面角 $/\left(^{\circ}\right)$} \\
\hline \multicolumn{3}{|c|}{$[\mathrm{C}(6), \mathrm{C}(7), \mathrm{C}(8)]-[\mathrm{C}(13), \mathrm{C}(8), \mathrm{C}(9)]$} & 4.82 \\
\hline \multicolumn{3}{|c|}{$[\mathrm{C}(11), \mathrm{C}(14), \mathrm{C}(15)]-[\mathrm{C}(10), \mathrm{C}(11), \mathrm{C}(12)]$} & 2.10 \\
\hline \multicolumn{3}{|c|}{$[C(9), C(10), C(21)]-[C(22), C(23), C(28)]$} & 0.38 \\
\hline
\end{tabular}

从图中可以看出, 中间体 $\mathbf{3}$ 在 $350 \mathrm{~nm}$ 处呈现一个中 等强度的吸收峰, 而在 $250 \sim 300 \mathrm{~nm}$ 之间呈现很弱的吸 收. 随着中间体 3 上的溴原子被不同的共轭体所取代, 目标化合物 $4 \mathbf{a} \sim \mathbf{4 d}$ 在 $250 \sim 325 \mathrm{~nm}$ 之间的吸收逐渐增 强, 这主要是由于分子的共轭体增大, 导致由 $\pi \rightarrow \pi *$ 跃 迁的 $\mathrm{K}$ 带吸收更强，甚至能够淹没 350 400 nm 之间的 $\mathrm{B}$ 带. 当然这些跃迁吸收主要来自于 $\pi \rightarrow \pi^{*}$ 的跃迁及分 子内电荷转移跃迁(ICT) 吸收 ${ }^{[21]}$, 通过后面的含时密度 泛函理论(TDDFT) 得到证实. 比较目标分子 $\mathbf{4 a} \sim \mathbf{4 d}$, 其

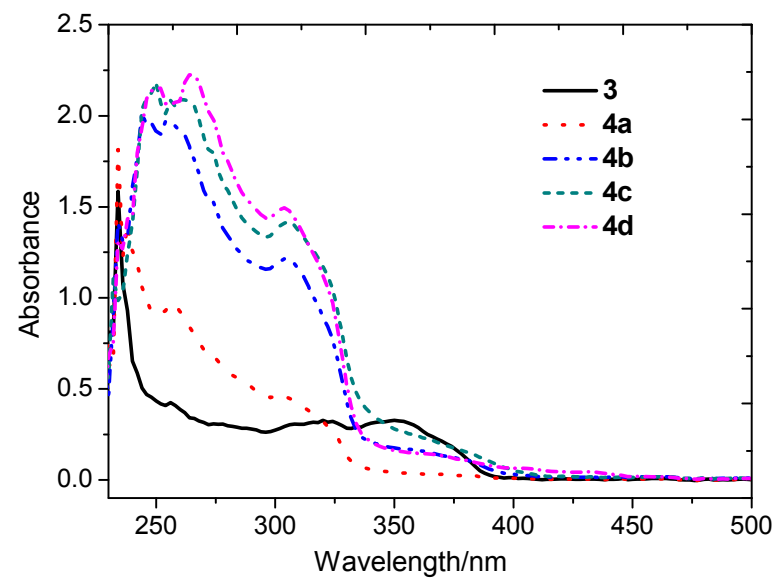

图 9 中间体 3 及目标化合物 $\mathbf{4 a} \sim \mathbf{4 d}$ 在 $\mathrm{CH}_{2} \mathrm{Cl}_{2}$ 溶液中的紫外 光谱图

Figure 9 UV-Vis absorption spectra of intermediate 3 and target compounds $\mathbf{4 a} \sim \mathbf{4 d}$ in $\mathrm{CH}_{2} \mathrm{Cl}_{2}$ solution

在 250 330 nm 之间的吸收呈现出相似的吸收峰型, 在 $350 \mathrm{~nm}$ 以后的矮而宽的吸收呈现出微弱的红移, 这主 要由于分子共轭体系的增大所致.

为了更好地理解系列目标化合物的电子跃迁性质， 我们对代表性目标化合物 $4 \mathrm{~b}$ 展开了密度泛函理论计算, 图 10 为目标化合物 $\mathbf{4 b}$ 主要跃迁设计的部分分子轨道图, 对应的主要跃迁及其归属见表 4. 从图 10 可以看出，该 化合物在紫外区域的跃迁分别来自于 $\mathrm{HOMO} \rightarrow \mathrm{LUMO}$ 、 $\mathrm{HOMO}-1 \rightarrow \mathrm{LUMO}$ 及 $\mathrm{HOMO}-2 \rightarrow \mathrm{LUMO}+1$, 主要归 属于 $\pi \rightarrow \pi^{*}$ 及分子内电荷转移(ICT) 跃迁吸收.

\subsection{2 荧光光谱分析}

为了更好了解系列化合物的发射光谱特点，我们对 其进行了苂光光谱实验，图 11 为中间体 $\mathbf{3}$ 及目标化合物 4a 4d 在 $\mathrm{CH}_{2} \mathrm{Cl}_{2}$ 溶液中的荧光光谱图. 从图中可以看 出, 中间体 $\mathbf{3}$ 在 $411 \mathrm{~nm}$ 处呈现出最强的苂光发射, 随着 中间体 3 苯环上的溴原子被苯乙炔基、五氟苯乙炔基、 菜乙炔基葱乙炔基取代分别得到 $\mathbf{4 a} \sim \mathbf{4 d}$, 原有的发射 峰分别成两个, 这可能由于共轭体系的增大, 使 $\pi-\pi$ 作 用增强，促使其形成激基缔合物. 这在另一方面说明它 们的堆积作用增强，从而使得荧光强度比中间体 3 要弱 ${ }^{[22]}$. 此外, 随着共轭体系的逐渐增大导致了它们的发射 峰有一个红移的倾向. 从图中可以看出化合物 $\mathbf{4 d}$ 在 500 $\mathrm{nm}$ 左右有一个较弱的发射峰, 因此该化合物在紫外灯 下 $(365 \mathrm{~nm})$ 呈现黄光. 从以上分析可以看出, 共轭体系 的增大有利于分子之间形成 $\pi-\pi$ 堆积，从而有望实现区 域性组装.

\section{2 结论}

通过经典的 Witting-Horner 反应及 Sonogashira 交叉 偶联反应以较高的收率合成了 4 个不对称型的氢键受体 


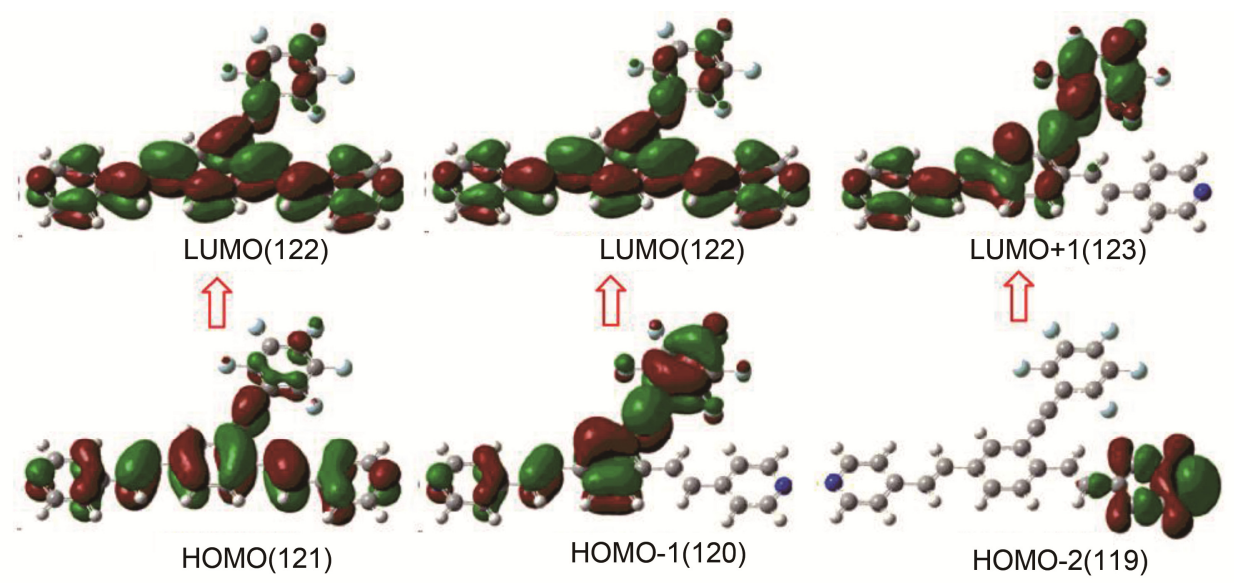

图 10 目标化合物 $4 \mathrm{~b}$ 主要跃迁涉及的部分分子轨道图

Figure 10 Selected frontier orbitals involved in the transition for target compound $\mathbf{4 b}$ Contour values $\left(0.04\left(\mathrm{e} / \mathrm{b}^{3}\right)^{1 / 2}\right.$

表 2 TDDFT 计算模型分子 $\mathbf{4 b}$ 主要的电子跃迁及其归属

Table 2 Major electronic excitations for $\mathbf{4 b}$ determined by TDDFT methods (B3LYP/6-31G ${ }^{*}$ )

\begin{tabular}{cccl}
\hline 波长 $/ \mathrm{nm}$ & 振子强度 $(f)$ & \multicolumn{1}{c}{ 跃迁 } & 归属 \\
\hline 457 & 0.9722 & HOMO $\rightarrow$ LUMO $(70 \%)$ & $\pi \rightarrow \pi^{*}$ \\
365 & 1.3586 & HOMO-1 $\rightarrow$ LUMO (52\%) & ICT \\
283 & 0.2134 & HOMO-2 $\rightarrow$ LUMO+1 $(37 \%)$ & ICT \\
\hline
\end{tabular}

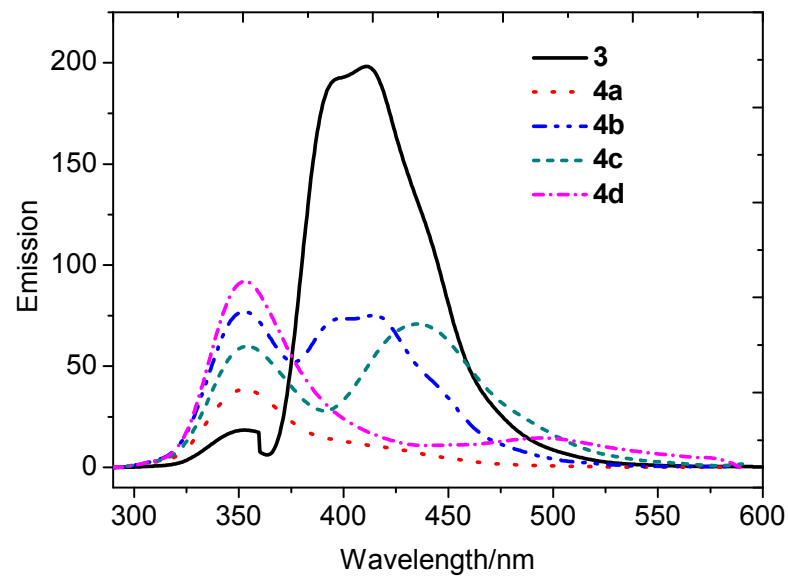

图 11 中间体 3 及目标化合物 $\mathbf{4 a} \sim 4 \mathrm{~d}$ 在 $\mathrm{CH}_{2} \mathrm{Cl}_{2}$ 溶液中的苂 光光谱图

Figure 11 Fluorescence emission spectrum of intermediate 3 and target compounds $\mathbf{4 a} \sim \mathbf{4 d}$ in $\mathrm{CH}_{2} \mathrm{Cl}_{2}$ solution

分子 1,4-双[2-(4-吡啶基)乙烯基]苯衍生物 4a $\sim \mathbf{4 d}$, 并对 其结构及性质进行了研究. 该系列分子结构均通过了核 磁、质谱及元素分析结构表征. 此外化合物 $\mathbf{4 b}$ 的结构通 过 $\mathrm{X}$ 射线单晶衍射确认. 从化合物 $\mathbf{4 b}$ 的晶体结构可以 看出 4-吡啶乙烯基在苯的同侧, 这主要与邻位上的芳环 乙炔基的空间效应有关. 四个氢键受体分子通过间苯二 酚组装, 然后光致环化, 去模版得到图 12 的结果.

紫外可见光谱结合 TDDFT 计算表明目标化合物 4a $\sim 4$ 在紫外区域均呈现出强烈的 $\pi \rightarrow \pi^{*}$ 及 ICT 跃迁吸 收, 完全符合这类化合物的吸收特征. 荧光光谱表明随 着共轭体系的逐渐增大, 它们的发射峰有一个红移的倾 向, 且有利于分子之间形成 $\pi-\pi$ 堆积. 以上结果将为后 续的氢键自组装的区域选择性及光照环化实验研究提 供一定的合成基础及理论依据.

\section{3 实验部分}

\section{1 仪器与试剂}

实验过程中大部分反应都是在氮气或者氩气保护 下进行的, 采用的是标准的 Schlenk 双排管真空线技术. 溶剂四氢呋喃经钠/二苯甲酮处理, 二氯甲烷和正已烷 用氢化钙处理，三乙胺用 $\mathrm{KOH}$ 干燥处理，使用前将处 理好的溶剂新蒸出并脱气. 试剂 2,5-二甲基溴苯、4-吡 啶甲醛、叔丁醇钾、碘化亚铜、亚磷酸三乙酯、三苯基 膦、溴苯、五氟代碘苯、 $\alpha$-溴代菜、9-溴萝等从中国医 药集团上海试剂公司购买，溶剂均为国产分析纯；2,5二溴甲基溴苯 ${ }^{[18]}$ 、苯乙炔 ${ }^{[19]}$ 、五氟苯乙炔 ${ }^{[19]} 、 \alpha$-乙炔基 䒺 ${ }^{[19]} 、 9$-乙炔基葱 ${ }^{[19]}$ 均按文献方法合成得到.

元素分析 $(\mathrm{C} 、 \mathrm{H} 、 \mathrm{~N})$ 经华中师大分析测试中心 Vario ElIII CHNSO 元素分析仪测得; 质谱(MS)使用 Firmigan Trace 质谱仪测定; ${ }^{1} \mathrm{H}$ NMR、 ${ }^{13} \mathrm{C}$ NMR 和 ${ }^{31} \mathrm{P}$ NMR 经 Varian MERCURY Plus $400 \mathrm{MHz}$ 或 Varian MERCURY Plus $600 \mathrm{MHz}$ 核磁共振仪测得, 其中 ${ }^{1} \mathrm{H}$ NMR、 ${ }^{13} \mathrm{C}$ NMR 谱化学位移以 TMS 作为内标, ${ }^{31} \mathrm{P}$ NMR 谱化学位 移以 $85 \% \mathrm{H}_{3} \mathrm{PO}_{4}$ 作为外标.

化合物的单晶在 Bruker Apex CCD X 射线衍射仪下 收集, 其晶体衍射数据经过 SAINT v 6.26 还原, 然后经 SADABS 软件修正晶胞参数. 晶体结构由直接法 (SHELXTL-97)程序解出. 以差值傅立叶合成法定出非 


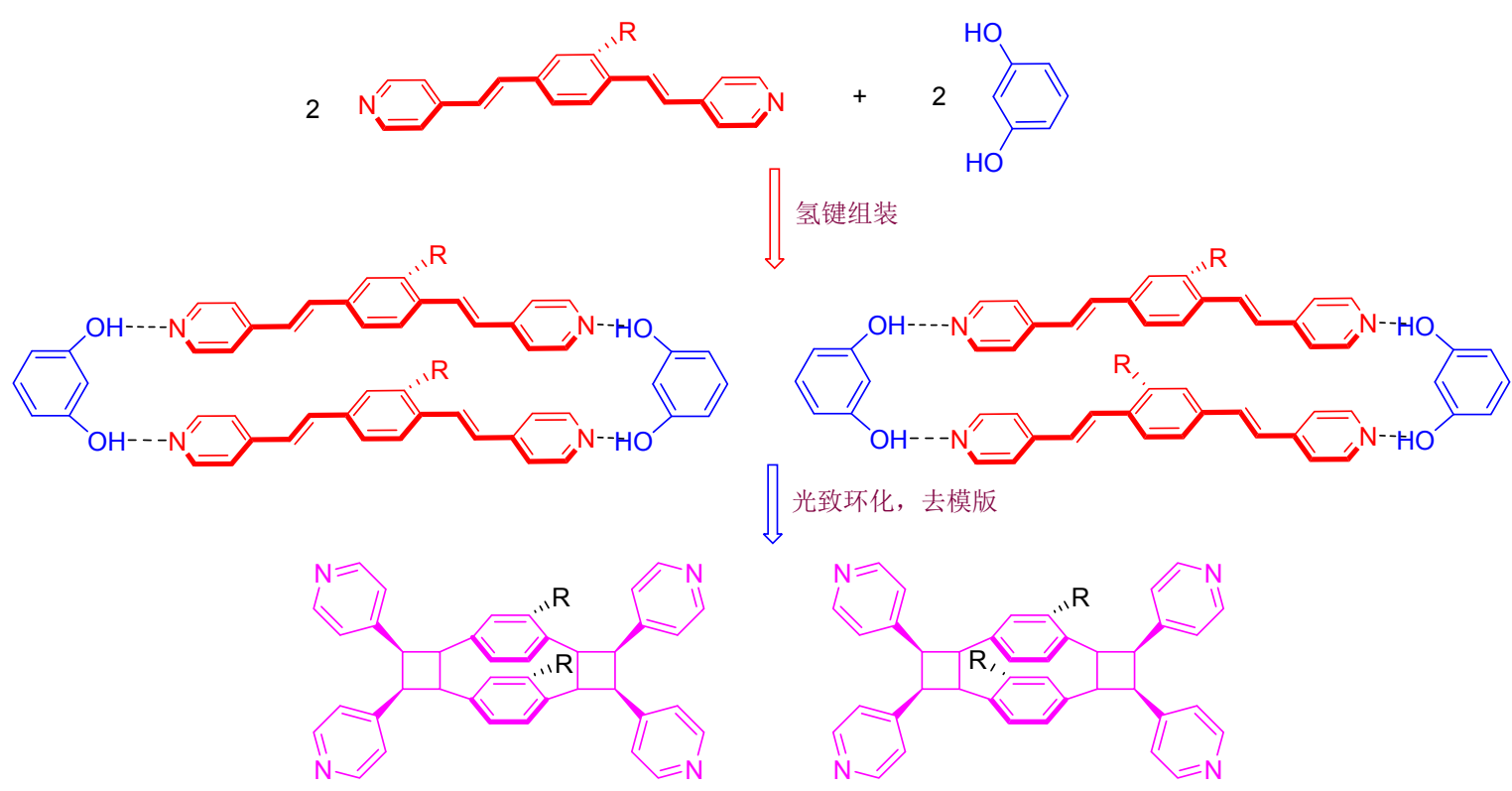

图 12 根据晶体结构分析实际过程中可能的氢键组装情况

Figure 12 Possible process of hydrogen-bond self-assemble according to crystal structure analysis

氢原子坐标, 并以各项异性热参数用于全矩阵最小二乘 法对其进行修正，氢原子用理论加氢的方式产生.

紫外可见光谱使用日本岛津公司生产的 UV-3600 紫外-可见-近红外光谱仪测定; 苂光光谱在 Fluoromax$P$ 苂光光谱仪(HORIBA JOBIN YVON INC)测定.

DFT 计算通过高斯 09 程序在 B3LYP/6-31G*理论水 平下进行; 几何优化在没有任何对称性限制下完成的, 频率计算基于几何优化结果; 电子跃迁来自于含时密度 泛函理论计算(TDDFT)结果; 分子轨道贡献及可视化分 别通过 Multiwfn2.6.1_bin_Win 软件包及 GaussView 5.0 程序完成.

\section{1 中间体 2-溴-1,4-二苯甲基膦酸二乙酯(2)的合成}

氮气保护下, 在带有搅拌磁子的 $50 \mathrm{~mL}$ 圆底烧瓶中 依次加入化合物 1 (1.6 g, $4.8 \mathrm{mmol})$ 、亚磷酸三乙酯 $(9$ $\mathrm{mL}$ ), 混合体系在 $140{ }^{\circ} \mathrm{C}$ 下加热回流 $4 \mathrm{~h}$ 后, 冷却至室 温, 加入 $30 \mathrm{~mL}$ 正己烷, 析出固体(在常温下易变成液 体), 减压蒸馏除去过量的亚磷酸三乙酯及澳乙烷, 得 到 $1.5 \mathrm{~g}$ 米白色粘稠固体, 产率 $70 \%$. m.p. $25 \sim 30{ }^{\circ} \mathrm{C} ;{ }^{1} \mathrm{H}$ NMR (400 MHz, $\left.\mathrm{CDCl}_{3}\right) \delta: 1.26\left(\mathrm{~s}, 12 \mathrm{H}, \mathrm{CH}_{3}\right), 3.09(\mathrm{~d}$, $\left.J=20 \mathrm{~Hz}, 2 \mathrm{H}, \mathrm{Ar}-\mathrm{CH}_{2}\right), 3.37$ (d, $J=20 \mathrm{~Hz}, 2 \mathrm{H}, \mathrm{Ar}-\mathrm{CH}_{2}$ ), 4.05 (s, 8H, $\left.\mathrm{CH}_{3}\right), 7.23$ (s, 1H, Ar-H), 7.41 (s, $\left.1 \mathrm{H}, \mathrm{Ar}-\mathrm{H}\right)$, 7.52 (s, $1 \mathrm{H}, \mathrm{Ar}-\mathrm{H}) ;{ }^{13} \mathrm{C} \mathrm{NMR}\left(100 \mathrm{MHz}, \mathrm{CDCl}_{3}\right) \delta: 15.94$, 15.99, $31.86\left(\mathrm{~d}, J=17 \mathrm{~Hz}, \mathrm{O}=\mathrm{PCH}_{2}\right), 33.25(\mathrm{~d}, J=17 \mathrm{~Hz}$, $\left.\mathrm{O}=\mathrm{PCH}_{2}\right), 61.81\left(\mathrm{OCH}_{2}\right), 61.87\left(\mathrm{OCH}_{2}\right), 124.39,128.56$, 130.07, 131.16, 132.04, 132.09, 133.57; MS m/z: 456.01 $\left(\mathrm{M}^{+}\right)$. Anal. calcd for $\mathrm{C}_{16} \mathrm{H}_{27} \mathrm{BrO}_{6} \mathrm{P}_{2}$ : C 42.03, H 5.95; found C 42.10, H 5.88 .

\section{2 中间体 2-溴-1,4-双[2-(4-吡啶基)乙烯基]苯(3)的合 成}

氮气保护下, 向含有磁子的 $50 \mathrm{~mL}$ 圆底烧瓶中加入 叔丁醇钾 $(381 \mathrm{mg}, 3.40 \mathrm{mmol})$, 经恒压滴液漏斗再往烧 瓶中逐渐滴加入含有中间体 $2(457 \mathrm{mg}, 1.00 \mathrm{mmol})$ 及 4吡啶甲醛 $(252 \mathrm{mg}, 2.35 \mathrm{mmol})$ 的四氢呋喃 $(\mathrm{THF})$ 溶液, 混合体系室温反应 $1 \mathrm{~h}$ 后, 向体系中滴加入 $\mathrm{HCl}(2$ $\mathrm{mol} / \mathrm{L}, 20 \mathrm{~mL}$ ), 蛋黄色沉淀析出, 然后加入饱和的 $\mathrm{NaHCO}_{3}$ 溶液, 最后体系用 $\mathrm{CH}_{2} \mathrm{Cl}_{2}$ 萃取, 有机相依次用 饱和 $\mathrm{NaHCO}_{3}$ 溶液, 饱和 $\mathrm{NaCl}$ 溶液洗涤, 无水 $\mathrm{Na}_{2} \mathrm{SO}_{4}$ 干燥, 旋干, 粗产品经柱层析分离[硅胶 200 300 目, $V(\mathrm{THF}): V($ 石油醚 $)=1 ： 2$ ]得到 $0.20 \mathrm{~g}$ 黄色固体, 产率 55\%. m.p. 192 $196{ }^{\circ} \mathrm{C} ;{ }^{1} \mathrm{H}$ NMR $\left(400 \mathrm{MHz}, \mathrm{CDCl}_{3}\right) \delta$ : $7.01(\mathrm{t}, J=16 \mathrm{~Hz}, 2 \mathrm{H}, \mathrm{CH}=), 7.20(\mathrm{~d}, J=16 \mathrm{~Hz}, 1 \mathrm{H}$, $\mathrm{CH}=), 7.38(\mathrm{dd}, J=8 \mathrm{~Hz}, 4 \mathrm{H}, \mathrm{Py}-\mathrm{H}), 7.49 \sim 7.52(\mathrm{~m}, 1 \mathrm{H})$, $7.68(\mathrm{t}, J=16 \mathrm{~Hz}, 2 \mathrm{H}, \mathrm{Py}-\mathrm{CH}=), 7.70(\mathrm{~s}, 1 \mathrm{H}), 8.58 \sim 8.62$ (m, 4H, Py-H); ${ }^{13} \mathrm{C}$ NMR (100 MHz, $\left.\mathrm{CDCl}_{3}\right) \delta: 120.79$, $120.95,124.93,126.02,126.95,127.64,128.82,130.71$, 130.99, 131.41, 135.72, 137.78, 143.74, 143.89, 150.21; MS $m / z$ : $362.02\left(\mathrm{M}^{+}\right)$. Anal. calcd for $\mathrm{C}_{20} \mathrm{H}_{15} \mathrm{BrN}_{2}$ : C 66.13, H 4.16; found C 66.20, H 4.02.

\section{3 目标化合物 $4 a \sim 4 d$ 的合成}

3.3.12-苯乙炔基-1,4-双[2-(4-吡啶基)乙烯基]苯(4b) 的合成

氮气保护下, 向含有磁子的 $25 \mathrm{~mL}$ 二颈瓶中依次加 入中间体 3 (100 mg, $0.275 \mathrm{mmol}), \mathrm{Pd}\left(\mathrm{PPh}_{3}\right)_{4}(32 \mathrm{mg}$, $0.028 \mathrm{mmol})$ 及 $\mathrm{CuI}(6 \mathrm{mg}, 0.041 \mathrm{mmol})$, 然后注入已脱 
气的 $\mathrm{THF}(10 \mathrm{~mL})$ 及 $\mathrm{Et}_{3} \mathrm{~N}(5 \mathrm{~mL})$, 室温搅拌 $30 \mathrm{~min}$ 后, 加入苯乙炔 $(140 \mathrm{mg}, 1.38 \mathrm{mmol})$, 体系加热回流反应 48 $\mathrm{h}$ 冷却至室温. 过滤(经硅藻土)得滤液, 旋干, 柱层析分 离 [硅胶 $200 \sim 300$ 目, $V$ (丙酮)： $V$ (石油醚) $=1 ： 1$ 作为洗 脱剂]得到 $68 \mathrm{mg}$ 黄色固体, 产率 64\%. m.p. 198 $203{ }^{\circ} \mathrm{C} ;{ }^{1} \mathrm{H}$ NMR $\left(400 \mathrm{MHz}, \mathrm{CDCl}_{3}\right) \delta: 7.08$ (d, $J=16 \mathrm{~Hz}$, $1 \mathrm{H}, \mathrm{CH}=), 7.16(\mathrm{~d}, J=16 \mathrm{~Hz}, 1 \mathrm{H}, \mathrm{CH}=), 7.27(\mathrm{~d}, J=16$ $\mathrm{Hz}, 1 \mathrm{H}, \mathrm{CH}=), 7.37 \sim 7.42(\mathrm{~m}, 7 \mathrm{H}), 7.52(\mathrm{~d}, J=8 \mathrm{~Hz}$, 1H), $7.59 \sim 7.61(\mathrm{~m}, 2 \mathrm{H}), 7.74(\mathrm{~d}, J=12 \mathrm{~Hz}, 2 \mathrm{H}), 7.90$ (d, $J=16 \mathrm{~Hz}, 1 \mathrm{H}, \mathrm{CH}=), 8.59 \sim 8.61(\mathrm{~m}, 4 \mathrm{H}) ;{ }^{13} \mathrm{C} \mathrm{NMR}(100$ $\mathrm{MHz}, \mathrm{CDCl}_{3}$ ) $\delta: 87.02(\mathrm{Ph}-\mathrm{C} \equiv), 95.19$ (Ar-C $\left.\equiv\right), 120.86$, $122.73,123.36,125.56,126.94,127.06,127.80,128.54$, $128.76,130.42,131.24,131.46,136.12,137.35,144.06$, 144.37, 150.21; MS m/z: $384.11\left(\mathrm{M}^{+}\right)$. Anal. calcd for $\mathrm{C}_{28} \mathrm{H}_{20} \mathrm{~N}_{2}$ : C 87.47, H 5.24; found C 87.23, H 5.36.

3.3 .2 2-五氟苯乙炔基-1,4-双[2-(4-吡啶基)乙烯基] 苯 $(4 b)$ 的合成

化合物 $4 \mathbf{b}$ 的合成步骤与化合物 $4 \mathbf{a}$ 相似, 投料量: 中间体 3 (100 mg, $0.275 \mathrm{mmol}), \mathrm{CuI}$ (6 mg, $0.24 \mathrm{mmol})$, $\mathrm{Pd}\left(\mathrm{PPh}_{3}\right)_{4}(0.28 \mathrm{~g}, 0.24 \mathrm{mmol})$, 三乙胺 $(5 \mathrm{~mL})$ 及四氢呋喃 $(10 \mathrm{~mL})$, 五氟苯乙炔 $(106 \mathrm{mg}, 0.55 \mathrm{mmol})$. 粗产品经柱 层析分离 [硅胶 200 300 目, $V$ (丙酮) $: V$ (石油醚 $)=1 ： 1$ 作为洗脱剂]得到 $101 \mathrm{mg}$ 紫黑色固体, 产率 78\%. m.p. 229 $233{ }^{\circ} \mathrm{C} ;{ }^{1} \mathrm{H}$ NMR (400 MHz, $\left.\mathrm{CDCl}_{3}\right) \delta: 7.11$ (d, $J=$ $16 \mathrm{~Hz}, 1 \mathrm{H}, \mathrm{CH}=), 7.19(\mathrm{~d}, J=16 \mathrm{~Hz}, 1 \mathrm{H}, \mathrm{CH}=), 7.30$ (d, $J=4 \mathrm{~Hz}, 1 \mathrm{H}), 7.42$ (dd, $J=4 \mathrm{~Hz}, 4 \mathrm{H}, \mathrm{Py}-\mathrm{H}), 7.63$ (d, $J=8$ $\mathrm{Hz}, 1 \mathrm{H}), 7.80$ (t, $J=8 \mathrm{~Hz}, 2 \mathrm{H}), 7.90$ (d, $J=16 \mathrm{~Hz}, 1 \mathrm{H}$, $\mathrm{CH}=), 8.62(\mathrm{t}, J=4 \mathrm{~Hz}, 4 \mathrm{H}, \mathrm{Py}-\mathrm{H}) ;{ }^{13} \mathrm{C} \mathrm{NMR}(100 \mathrm{MHz}$, $\left.\mathrm{CDCl}_{3}\right) \delta: 102.06$ (Ar-C $\left.\equiv\right), 115.89$ (Ar-C $\left.\equiv\right), 115.96$, $116.18,131.42,131.52,136.15,137.17,143.23,163.70$, 166.21, 173.81; MS m/z: $474.14\left(\mathrm{M}^{+}\right)$. Anal. calcd for $\mathrm{C}_{28} \mathrm{H}_{15} \mathrm{~F}_{5} \mathrm{~N}_{2}$ : C 70.89, H 3.19; found C 70.70, H 3.23.

3.3.32-(1-荟乙炔基)-1,4-双 [2-(4-吡啶基)乙烯基]苯 (4c) 的合成

化合物 $\mathbf{4 c}$ 的合成步骤与化合物 $4 \mathbf{a}$ 相似，投料量: 中间体 3 (100 mg, $0.275 \mathrm{mmol}$ ), CuI (6 mg, $0.24 \mathrm{mmol}$ ), $\mathrm{Pd}\left(\mathrm{PPh}_{3}\right)_{4}(0.28 \mathrm{~g}, 0.24 \mathrm{mmol})$, 三乙胺 $(5 \mathrm{~mL})$ 及四氢呋 喃(10 mL), 1-乙炔基菜 $(83 \mathrm{mg}, 0.55 \mathrm{mmol})$. 粗产品经柱 层析分离 [硅胶 200 300 目, $V$ (丙酮)：V(石油醚 $)=1 ： 1$ 作为洗脱剂]得到 $83 \mathrm{mg}$ 亮黄色固体, 产率 69\%. m.p. 256 260 ${ }^{\circ} \mathrm{C} ;{ }^{1} \mathrm{H}$ NMR (400 MHz, $\left.\mathrm{CDCl}_{3}\right) \delta: 7.10$ (d, $J=$ $20 \mathrm{~Hz}, 1 \mathrm{H}, \mathrm{CH}=), 7.18$ (d, J=16 Hz, 1H, CH=), 7.29 (d, $J=4 \mathrm{~Hz}, 1 \mathrm{H}), 7.41$ (dd, $J=4 \mathrm{~Hz}, 4 \mathrm{H}, \mathrm{Py}-\mathrm{H}), 7.50 \sim 7.59$ (m, 4H), 7.77 (d, $J=12 \mathrm{~Hz}, 1 \mathrm{H}), 7.83$ (d, $J=8 \mathrm{~Hz}, 2 \mathrm{H})$, $7.91(\mathrm{~d}, J=8 \mathrm{~Hz}, 2 \mathrm{H}), 8.06$ (d, $J=16 \mathrm{~Hz}, 1 \mathrm{H}, \mathrm{CH}=), 8.44$ $(\mathrm{d}, J=8 \mathrm{~Hz}, 1 \mathrm{H}), 8.60(\mathrm{~s}, 4 \mathrm{H})$. 由于在常用忥代试剂中溶 解度较差, 没有收集到该化合物的碳谱. MS m/z: 434.20 $\left(\mathrm{M}^{+}\right)$. Anal. calcd for $\mathrm{C}_{32} \mathrm{H}_{22} \mathrm{~N}_{2}$ : C 88.45, H 5.10; found C 88.39, H 5.31.

3.3 .4 2-(9-葱乙炔基)-1,4-双[2-(4-吡啶基)乙烯基]苯 (4d) 的合成

化合物 4d 的合成步骤与化合物 $4 \mathbf{a}$ 相似, 投料量: 中间体 3 (200 mg, $0.45 \mathrm{mmol}$ ), CuI (12 mg, $0.48 \mathrm{mmol}$ ), $\mathrm{Pd}\left(\mathrm{PPh}_{3}\right)_{4}(0.56 \mathrm{~g}, 0.48 \mathrm{mmol})$, 三乙胺 $(10 \mathrm{~mL})$ 及四氢呋 喃(20 mL), 9-乙炔基蒽(182 mg, $0.90 \mathrm{mmol})$. 粗产品经 柱层析分离[硅胶 $200 \sim 300$ 目, $V$ (丙酮)： $V$ (石油醚 $)=$ $1: 1$ 作为洗脱剂]得到 $175 \mathrm{mg}$ 黄色固体, 产率: $66 \%$. m.p. 300 303 ${ }^{\circ} \mathrm{C} ;{ }^{1} \mathrm{H}$ NMR (400 $\left.\mathrm{MHz}, \mathrm{CDCl}_{3}\right) \delta: 7.14$ $(\mathrm{d}, J=16 \mathrm{~Hz}, 1 \mathrm{H}, \mathrm{CH}=), 7.21(\mathrm{~d}, J=16 \mathrm{~Hz}, 1 \mathrm{H}, \mathrm{CH}=)$, $7.34(\mathrm{~d}, J=16 \mathrm{~Hz}, 1 \mathrm{H}, \mathrm{CH}=), 7.43(\mathrm{dd}, J=4 \mathrm{~Hz}, 4 \mathrm{H}$, Py-H), $7.54 \sim 7.61(\mathrm{~m}, 5 \mathrm{H}), 7.83(\mathrm{~d}, J=8 \mathrm{~Hz}, 1 \mathrm{H}), 7.95$ (s, $1 \mathrm{H}), 8.06 \sim 8.07(\mathrm{~m}, 2 \mathrm{H}), 8.20(\mathrm{~d}, J=16 \mathrm{~Hz}, 1 \mathrm{H}, \mathrm{CH}=)$, $8.50(\mathrm{~s}, 1 \mathrm{H}), 8.59 \sim 8.67(\mathrm{~m}, 6 \mathrm{H})$. 由于在常用氝代试剂 中溶解度较差, 没有收集到该化合物的碳谱. MS m/z: $484.23\left(\mathrm{M}^{+}\right)$. Anal. calcd for $\mathrm{C}_{36} \mathrm{H}_{24} \mathrm{~N}_{2}$ : C 89.23, H 4.99; found $\mathrm{C} 89.30, \mathrm{H} 4.92$.

辅助材料(Supporting Information) 最终产物和部分 中间体的 ${ }^{1} \mathrm{H}$ NMR 及部分 ${ }^{13} \mathrm{C} \mathrm{NMR}$ 谱图，化合物 $\mathbf{4 b}$ 的 晶体数据, 以及化合物 $\mathbf{4 a}$ 和 $\mathbf{4 c}$ 经 DFT 方法(B3LYP/ $\left.6-31 \mathrm{G}^{*}\right)$ 优化下的构型. 这些材料可以免费从本刊网站 (http://sioc-journal.cn/)上下载.

\section{References}

[1] Tozawa, T.; Jones, J. T. A.; Swamy, S. I.; Jiang, S.; Adams, D. J.; Shakespeare, S.; Clowes, R.; Bradshaw, D.; Hasell, T.; Chong, S. Y.; Tang, C.; Thompson, S.; Parker, J.; Trewin, A.; Bacsa, J.; Slawin, A. M. Z.; Steiner, A.; Cooper, A. I. Nat. Mater. 2009, 8, 973.

[2] Yan, X.; Xu, D.; Chi, X.; Chen, J.; Dong, S.; Ding, X.; Yu, Y.; Huang, F. Adv. Mater. 2012, 24, 362.

[3] Hu, Q. D.; Tang, G. P.; Chu, P. K. Acc. Chem. Res. 2014, 47, 2017.

[4] Kuehl, C. J.; Huang, S. D.; Stang, P. J. J. Am. Chem. Soc. 2001, 123, 9634.

[5] Ensslen, P and Wagenknecht, H. Acc. Chem. Res., 2015, 48, 2724.

[6] Koshti, V. S.; Mote, N. R.; Gonnade, R. G. and Chikkali, S. H. Organometallics 2015, 34, 4802.

[7] Elemans, J. A. A. W.; Slangen, R. R. J.; Rowan, A. E.; Nolte, R. J. M. J. Org. Chem., 2003, 68, 9040.

[8] MacGillivray, L. R.; Papaefstathiou, G. S.; Friscic, T.; Hamilton, T. D.; Bucar, D-K.; Chu Q.; Varshney, D. B.; Georgiev, I. V. Acc. Chem. Res. 2008, 41, 280.

[9] Sokolov, A. N.; Friscic, T.; MacGillivray, L. R. J. Am. Chem. Soc. 2006, 128, 2806.

[10] Sokolov, A. N.; Bucar, D-K.; Baltrusaitis, J.; Gu, S. X.; MacGillivray, L. R. Angew. Chem. 2010, 122, 4369. 
[11] MacGillivray, L. R. J. Org. Chem. 2008, 73, 3311.

[12] Friscic, T.; Drab, D. M.; MacGillivray, L. R. Org. Lett. 2004, 6, 4647.

[13] Frišcic, T.; MacGillivray, L. R. Chem. Commun. 2003, 1306.

[14] Jiang, B.; Lei, Y.; Zhao, X.-L. J. Org. Chem. 2008, 73, 7833.

[15] Lahann, J.; Höcher, H.; Langer, R. Angew. Chem., Int. Ed. 2001, $40,726$.

[16] Sillen, C.; Liu, N.; Ho, W.; Maddox, J. B.; Mukamel, S.; Liu, B.; Bazan, G. C. Nano Lett. 2008, 8, 208.

[17] Bolm, C.; Whelligan, D. K. Adv. Synth. Catal. 2006, 348, 2093.
[18] Kay, K.-Y.; Baek, Y. G. Chem. Ber. /Recl. 1997, 130, 581.

[19] Wu, X. H.; Jin, S.; Liang, J. H.; Li, Z. Y.; Yu, G.-A.; Liu, S. H. Organometallics 2009, 28, 2450.

[20] Wang, L.; Tao, X-L.; Yang, J-X.; Yu, W-T.; Ren, Y.; Xin, Y.; Liu, Z.; Jiang, M-H. J Solid State Chem. 2004, 177, 4293.

[21] Kaminker, R.; Lahav, M.; Motiei, L.; Vartanian, M.; Popovitz-Biro, R.; Iron, M. A.; vander Boom, M. E. Angew. Chem., Int. Ed. 2010, 49, 1218.

[22] Hrobarikova, V.; Hrobarik, P.; Gajdos, P.; Fitilis, I.; Fakis, M.; Persephonis, P.; Zahradnık, P. J. Org. Chem. 2010, 75, 3053.

(Li, L.; Lu, Z.) 